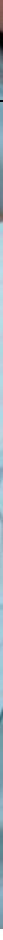

Pneumokokkenpneumonien

\title{
Effektiv vorbeugen ist besser als heilen
}

\begin{abstract}
Die ambulant erworbene Pneumonie ist die akute Infektionskrankheit mit der höchsten Sterblichkeit weltweit, Tendenz vor allem bei alten Menschen steigend. In bis zu 50\% der Fälle sind Pneumokokken die Ursache. Da bei der Diagnostik und Therapie mittelfristig keine wesentlichen Verbesserungen absehbar sind, ist die Impfung von Risikogruppen für die Mortalitätssenkung so immens wichtig. Aber diesbezüglich liegt in Deutschland einiges im Argen.
\end{abstract}

Die ambulant erworbene Pneumonie ist weiterhin die akute Infektionskrankheit mit der global höchsten Sterblichkeit. Die Zahl von Pneumonien wird in den nächsten Jahren aufgrund der weltweit zunehmenden Lebenserwartung weiter steigen, weil die Pneumonie klassischerweise die Erkrankung des älteren Menschen ist [1]. Pneumokokken verursachen 30-50\% der ambulant erworbenen Pneumonien und sind damit nach wie vor der wichtigste Erreger bei dieser Erkrankung [2]. Eine gerade publizierte Studie geht von knapp 25000 stationär behandelten Patienten mit Pneumokokkenpneumonie in Großbritannien (UK) aus [3]. Bei einer Sterblichkeit dieser Patienten von knapp 20\% bedeutet das fast 5000 Todesfälle im Jahr alleine durch diese Form der Pneumonie. Zum Vergleich starben 2014 in UK 1807 Patienten bei Verkehrsunfällen.

\section{Pneumokokken sind stark pathogen}

Die Sterblichkeit bei Pneumokokkenpneumonie hat sich in den letzten Jahrzehnten praktisch nicht verändert. Dabei spielt, anders als bei anderen Infektionen, die Resistenzentwicklung keine Rolle. Pneumokokken sind in Deutschland gegenüber allen empfohlenen antibiotischen Substanzen sensibel. Wesentlicher Grund für die hohe Sterblichkeit sind verschiedene Pathogenitätsfaktoren der Pneumokokken, die einerseits die Invasivität der Erreger und damit eine bakteriämische Infektion begünstigen und andererseits das Ausmaß des Gewebeschadens in der Lunge fördern. Daher ist es ist absehbar, dass weder Fortschritte in der Diagnostik noch neue Antibiotika ein Erhöhung der
Überlebensraten mit sich bringen werden. Langfristige Strategien zielen auf eine Verbesserung der Abwehrkraft der Patienten durch Modulation der Immunantwort. Erste Ergebnisse sind aber nicht vor Ablauf weiterer 10 Jahre zu erwarten.

\section{Direkt umsetzbaren Schutz bietet nur die Impfung}

Die einzige direkt umsetzbare Maßnahme, die zu einer Verringerung der Rate an Pneumokokkenpneumonien beitragen kann, ist daher das Vermeiden der Erkrankung durch Impfen.

Die Pneumokokkenkonjugatimpfung im Kleinkindesalter (624 Monate) hat sich im letzten Jahrzehnt zu einer Erfolgsgeschichte entwickelt. Bis 2005 war die invasive Pneumokokkenerkrankung die am häufigsten zum Tode führende Krankheit bei Kindern. Seit Einführung der Impfung - in Deutschland 2006 - hat sich die Zahl dieser Erkrankungen im Kindesalter in allen westlichen Ländern auf unter 10\% reduziert [4]. Neben dem Effekt auf die Rate an Pneumokokkenerkrankungen im Kindsalter gab es jedoch einen zusätzlichen überraschenden Effekt bei Erwachsenen. Auch da nahm die Rate an Pneumokokkenerkrankungen deutlich ab, der sogenannte Herdeneffekt. Kinder sind häufig chronisch mit Pneumokokken kolonisiert und übertragen diese auf die Erwachsenen. Der im Kindesalter verwendete Pneumokokkenkonjugatimpfstoff erzeugt eine mukosale Immunität, die eine Besiedlung verhindert und damit auch die Übertragung ausschließt.

Trotz des Herdenimmunitätseffekts ist die Rate an Pneumokokkenerkrankungen im Erwachsenenalter weiter hoch. Eine 
Pneumokokkenimpfung wird daher seit Jahren auch für ältere Menschen (>60 Jahre), Menschen mit chronischen Erkrankungen (insbesondere mit chronischen Lungenerkrankungen) und immunsupprimierte Patienten empfohlen. Im Gegensatz zur mit $85 \%$ hohen Durchimpfungsrate im Kindesalter ist die Impfrate im Erwachsenenalter mit weniger als $10 \%$ bei Patienten, für die eine Empfehlung zur Impfung ausgesprochen wurde, sehr gering. Neben der generellen, überwiegend ideologisch motivierten Impfskepsis in Deutschland, dürfte ein wesentlicher Grund in der insgesamt niedrigen Effektivität des im Erwachsenenalter eingesetzten Polysaccharidimpfstoffs liegen. Dieser Impfstoff erzeugt im Unterschied zum für Kinder empfohlenen Konjugatimpfstoff keine mukosale Immunität, sodass zwar Pneumokokkenbakteriämien vermieden werden können, die Pneumonierate aber praktisch nicht beeinflusst wird. Zudem ist die Immunantwort auf diesen Impfstoff gerade bei Älteren schwach. Zu diesem Ergebnis kamen alle Metaanalysen, in denen die randomisierten Studien zur Wirksamkeit des Polysaccharidimpfstoffs im Erwachenenalter im Hinblick auf die Pneumonierate ausgewertet wurden [5]. Bei immunsupprimierten Patienten, z.B. bei Patienten mit HIV-Infektion war sogar ein negativer Effekt der Polysaccharidimpfung zu beobachten [6].

\section{Konjugatimpfstoff vielerorts auch für Ältere empfohlen} Es stellte sich folgerichtig die Frage, ob der im Kindesalter so erfolgreiche Pneumokokkenkonjugatimpfstoff nicht auch eine bessere Alternative im Erwachsenenalter ist. Immunogenitätsdaten zeigten eine deutliche bessere Antikörperproduktion des Konjugatimpfstoffs gegenüber dem Polysaccharidimpfstoff [7]. Die erste randomisiert kontrollierte Studie, in der der 13valente Konjugatimpfstoff gegen Placebo bei mehr als 80000 Menschen in den Niederlanden getestet wurde, zeigt eine Reduktion der Pneumokokkenbakteriämierate um 75\% und der Pneumokokkenpneumonierate um 45\% für die im Impfstoff enthaltenden 13 Pneumokokkenserotypen [8]. Die Konjugatimpfung wurde von den verantwortlichen Gesundheitsbehörden daraufhin in den meisten europäischen Ländern und in den USA empfohlen [9].

Aus diesem Hintergrund verwundert es sehr, dass die Ständige Impfkommission (STIKO) in Deutschland die Pneumokokkenkonjugatimpfung weiterhin nur für spezielle Patientengruppen (nach Splenektomie und bei Immunsupprimierten), nicht aber generell für alle Risikogruppen empfiehlt [10]. Ein wesentlicher Grund dafür ist das Phänomen des sogenannten Serotypenshifts. Bedingt durch die hohe Effektivität der Konjugatimpfung im Kindealter reduziert sich die Zahl der im Impfstoff enthaltenen Serotypen, andere Serotypen nehmen diesen Platz ein. Dies war bereit nach Einführung des 7valenten Impfstoffs 2006 für die darin enthaltenen 7 Serotypen zu beobachten. Mit Neueinführung des 13valenten Konjugatimpfstoffs 2011 wurden die wichtigsten 6 neuen Serotypen im Impfstoff erfasst.

Auch für diese ist aber inzwischen wieder ein Serotypenshift zu beobachten. Damit könnte, bedingt durch den Herdeneffekt, die Effektivität des Impfstoffs im Erwachsenalter stark sinken.

\section{Sind wir in Deutschland rückständig?}

Ich hatte vor kurzem die Möglichkeit mit Michael Moore vom Center of Disease Control in Atlanta zu sprechen, einem der Verantwortlichen für die amerikanischen Empfehlungen. Die Nüchternheit der Amerikaner, frei von den bei uns üblichen ideologischen und wissenschaftstheoretischen Erwägungen ge- prägten Debatten, hat mich beeindruckt. Die Zahl der Pneumokokkenpneumonien in USA sei trotz des Serotypenshifts und des Herdeneffekts und trotz einer jahrezehntelangen Impfempfehlung für den Polysaccharidimpfstoff so hoch, dass der Einsatz von Konjugatimpfstoff kosteneffektiv wäre. Man müsse diese Entscheidung anhand der epidemiologischen Daten jedoch jedes Jahr prüfen. Das deckt sich mit den Zahlen aus Großbritannien, die zeigen, dass mehr als 2000 Todesfälle verhindert werden können, wenn sich die Konjugatimpfung durchsetzt [11].

Wir geben in Deutschland für viele Dinge viel Geld aus, ohne dass die Wirksamkeit der Maßnahmen nachgewiesen wäre. Für die Pneumokokkenkonjugatimpfung haben wir eindeutige Zahlen, die die Wirkung belegen, trotzdem kommt die STIKO nicht zu einer Empfehlung. Manchmal beneide ich die Amerikaner um ihren Pragmatismus.

\section{Literatur:}

1. GBD 2013 Mortality and Causes of Death Collaborators. Global, regional, and national age-sex specific all-cause and cause-specific mortality for 240 causes of death, 1990-2013: a systematic analysis for the Global Burden of Disease Study 2013. Lancet. 2015 Jan 10;385(9963):117-71.

2. Welte T, Torres A, Nathwani D. Clinical and economic burden of community-acquired pneumonia among adults in Europe. Thorax. 2012 Jan;67(1):71-9.

3. Rodrigo C, Bewick T, Sheppard C et al. Impact of infant 13-valent pneumococcal conjugate vaccine on serotypes in adult pneumonia. Eur Respir J. 2015 Jun;45(6):1632-41.

4. Waight PA, Andrews NJ, Ladhani SN et al. Effect of the 13-valent pneumococcal conjugate vaccine on invasive pneumococcal disease in England and Wales 4 years after its introduction: an observational cohort study. Lancet Infect Dis. 2015 May;15(5):535-43.

5. Moberley S, Holden J, Tatham DP, Andrews RM. Vaccines for preventing pneumococcal infection in adults. Cochrane Database Syst Rev. 2013 Jan 31;1:CD000422.

6. French N, Nakiyingi J, Carpenter LM et al. 23-valent pneumococcal polysaccharide vaccine in HIV-1-infected Ugandan adults: double-blind, randomised and placebo controlled trial. Lancet. 2000 Jun 17;355(9221):2106-11

7. Paradiso PR. Pneumococcal conjugate vaccine for adults: a new paradigm. Clin Infect Dis. 2012 Jul;55(2):259-64

8. Bonten MJ, Huijts SM, Bolkenbaas M et al. Polysaccharide conjugate vaccine against pneumococcal pneumonia in adults. N Engl J Med. 2015 Mar 19;372(12):1114-25

9. Tomczyk S, Bennett NM, Stoecker C et al. Centers for Disease Control and Prevention (CDC). Use of 13-valent pneumococcal conjugate vaccine and 23-valent pneumococcal polysaccharide vaccine among adults aged $\geq 65$ years: recommendations of the Advisory Committee on Immunization Practices (ACIP). MMWR Morb Mortal Wkly Rep. 2014 Sep 19;63(37):822-5

10. Empfehlungen der Ständigen Impfkommission (STIKO) am Robert Koch-Institut/Stand: August 2015; DOI 10.17886/EpiBull-2015-001

11. Welte T. Prevention is better than cure: time to change the focus of community-acquired pneumonia management. Eur Respir J. 2015 Jun;45(6):1524-5.

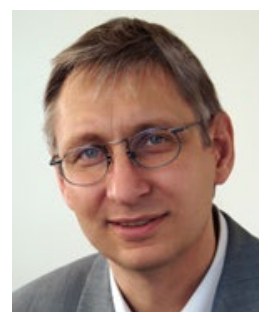

Prof. Dr. med. Tobias Welte

Klinik für Pneumologie

Medizinische Hochschule Hannover

Carl-Neuberg-Str. 1, 30625 Hannover

Welte.tobias@mh-hannover.de 